\title{
The pitfalls of a tool-based science and the promise of a problem-focused science
}

\author{
Patrick E. McKnight \\ George Mason University \\ Patrick McGovern \\ Buffalo State University
}

\author{
Michael J ohns \\ New York City Department of \\ Health and Mental Hygiene \\ J ulius Najab \\ George Mason University
}

\begin{abstract}
Our present social sciences are at risk of losing sight of their primary purpose: the goal of reducing uncertainty. For years social scientists have drifted slowly toward the routine of employing of accepted methodological, conceptual, and analytical tools rather than engaging in problem oriented inquiry. Scientific contributions are reviewed in accordance to their compliance with the routine application of tools rather than focusing on their ability to problem-solve for a wider population. Researchers in every area of psychology for instance now insist on using methods such as random assignment and control groups, as well as data analytic procedures such as null hypothesis significance testing without regard to their relevance. A problem-focused inquiry would not dictate the routine use of any particular tool but rather the judicious application of tools when deemed appropriate. The following article describes the current situation in the framework contrasting toolbased and problem-focused inquiry and offers several insights that may create a more balanced and fruitful approach to scientific inquiry.
\end{abstract}

Keywords: Scientific Progress, Methods, Problem Solving, Innovation

Over the past century, social science has come generally to be identified by its practitioners and other observers as a collection of various "disciplines" organized around the systematic study of the human race and its wide array of behavior, both on the individual and group levels. It is telling that this identity should come to be associated with the idea of "discipline," because the word's original meaning had much more to do with "conversion," "teaching," or "understanding" than today's definition of the word as "adherence," "following," "rules," or "punishment." Indeed, anyone who completed a social science graduate degree would recognize immediately the word in its modern guise, particularly its implications of adherence. But such an identity has come at a cost to science. While much is made of science as a method - a method that surely requires adherence to various norms and procedures - science ought to be thought of primarily as a means of reducing uncertainty about the world. Science is a means of engaging the fundamental problems that we face as humans when making both individual and collective decisions concerning our existence. The focus of social science's identity merely as a discipline shifts science's aims away from problem-solving and issues of uncertainty to the 
narrower activities of applying technique, following rules and adhering to routine.

The need for routines or the often necessary discussions about technique are not the targets of our critique. The problem as it is outlined here is that the discussion regarding the purpose or overall aim of research has often been sacrificed in order to stay within the boundaries of the accepted practices of any particular discipline. A tremendous amount of research in social science now conforms to a rigid routine involving a limited set of tools, rules, and techniques rather than questioning the type of tool required for solving the problem at hand. For example, many social scientists typically test theories by using laboratory studies conducted on college undergraduate students. These students generally complete selfreport measures that are then assessed by means of the "analysis of variance" (ANOVA) procedure, and then the results are reported in a highly structured format, usually to members of a very limited, selfselected sub-discipline within the social sciences. While not all social scientists employ these exact methods, this routine typifies the vast majority of research within any number of the social science subdisciplines.

It is this type of programmatic routinizing that causes concern. ${ }^{1}$ While necessary for the replication and standardization of methods and knowledge, routines can often inhibit progress, particularly when norms and procedures are followed blindly or out of disciplinary habit. For some time now, psychologists have recognized for some time now that behavior may persist in spite of contrary knowledge. Asch's recognition of this issue in his research on conformity (1956) pioneered research programs in judgment and decision-making (Goldstein \& Hogarth, 1997). Such research has borne out that even though data analysts are prone to routine compliance even when they may know the routine to be incorrect, as when, for example, Rosnow and Rosenthal (1995) and others (Zuckerman, Hodgins, Zuckerman, \& Rosenthal, 1993) observed the routine and inappropriate interpretation of interaction effects by trained analysts. Approximately one-third of the respondents in these surveys reported that their incorrect interaction interpretations were based upon what they were taught. Thus, learned routines might be positive (i.e., the two-thirds who accurately interpret the interaction) or negative (i.e., those inaccurately interpreting interactions). The point is that social scientists frequently follow routines endorsed by their sub-disciplines that may lead to erroneous conclusions. Rather than using the most appropriate methods or tools for critical inquiry of a particular problem or issue, investigators

${ }^{1}$ The term "routine" to which we refer includes research methods, data analytic procedures, and interpretative heuristics and their employment regardless of their relevance to the problem at hand. The routine might even be in the interpretation of these methods and procedures themselves. 
use what is most commonly employed by their respective discipline or what is mandated by the editorial boards of the all-important peer-review journals that function at the heart of all the social sciences. However, this compliance to routine may not only lead to erroneous conclusions but also may adversely affect our progress in seeking the reduction of uncertainty.

\section{Slow progress due to a tool-based approach?}

Our analysis of the relationship between the routine use of tools and the progress of social science rests on the assumption that the basis for science is to reduce uncertainty. What we consider a gain in knowledge represents the residue of our decreased uncertainty. When we remain equally uncertain about the phenomena we study despite our methods, we fail to make scientific progress and thus fail to increase knowledge. All areas of science work with this implicit goal; however, not every effort in science leads to a substantial decrease in uncertainty. Most studies ought to be designed and conducted to provide the greatest information gain (i.e., the greatest decrease in uncertainty). Assuming that social science has essentially come to be a tool-based endeavor, the consequences of this approach may be detrimental to such progress. From the perspective of uncertainty reduction, a tool-based approach may limit the types of questions, the methods of study and the procedures for analyzing the more important vexing problems and questions. Not only does a tool-based approach limit these aspects but a tool-based approach may slow or even arrest progress in social science.

Historically social science's primary focus has not been its tools. As in the case of psychology, social science focused instead on many rudimentary problems - both applied and basic - and, as seen in psychology's early efforts, it was directed at solving these problems. For example, the rudiments of learning, memory, and perception were all topics of general psychology in the early 20th century. In particular, perception attracted the greatest effort early on as the area in which researchers could make great contributions to our understanding of how people see and hear stimuli. Through constant debate and data/model comparisons, several major findings survived the first tests of critical scrutiny and have since withstood additional scrutiny, reduced our uncertainty about human behavior and thus became understood as "laws" (e.g., Steven's power law; gestalt laws of proximity, good continuation, and common fate). Without continual tests and challenges, the original findings would not have generated the necessary evidentiary strength fitting for the label "lawful." The methods employed in these research efforts varied greatly; and frequently the researcher would devise new tools to help solve the problem. Nowhere is there any evidence that every early researcher used the exact same design, experimental procedure, or 
statistical analysis. In fact, the evidence points overwhelmingly toward the idea that no single set of tools existed to test findings.

Several contemporary researchers have noted a stark contrast between past and present achievements in light of these tools, methods, and routines, and have concluded that progress in psychology has been slow compared to other areas of scientific inquiry. Most notably, Meehl (1978) attributed slow progress to the problems inherent in an over-reliance on null hypothesis significance testing (NHST). Cohen (1962), likewise adopted a stance regarding the impact of statistical routines when he addressed the concept of statistical power.

While we agree with both Meehl and Cohen's assertions regarding the pace of progress, their critique seems incomplete. We expand the list of problems limited to statistical procedures by including all routine practices, and attribute a tremendous amount of "slow growth" to the mechanistic and baseless applications of methodological tools. Meehl and Cohen provided just two explanations for why progress in psychology has been slow. But we challenge the reader to think of any area within psychology where great progress in the last 100 years has revolutionized our thinking about psychology. Behaviorism might come close but even the generalizability limits to these laboratory findings are unknown to this day (cf. representative design, Brunswik, 1956).

Understanding why early psychologists (pre-1980's) were able to make such significant contributions and later ones (post-1980's) were not necessitates a discussion of the role extant knowledge (in the form of research) plays in selecting one method over another. It appears that the founding thinkers in psychology used a variety of methods simply because they knew little about the phenomena they were studying, and they simply needed to start somewhere. Given their great uncertainty about phenomena and their even greater uncertainty about the "appropriate" methods to employ, the early psychologists were free to follow the problems presented by a phenomenon and not the methodological zeitgeist dictated by a discipline. However, as data accumulate, phenomena become associated with particular methods, and it became easier to justify the repeated use of one tool.

Research on cognitive dissonance provides us with an example of repeated tool use. After the publication by Festinger and Carlsmith (1959), researchers studied dissonance in a variety of ecologically meaningful situations (e.g., initiations, difficult decisions, punishment). Out of these studies came accepted routines. One such routine is assigning introductory psychology students to write an essay promoting a tuition increase. This exercise has become almost interchangeable with the dissonance phenomenon.

Perhaps the most insightful and craftily written design enhancements came from social psychology, yet these developed before the era of the 
disciplines' focus on tools. The most intriguing findings came from the designs and methods that fall outside what is now the standard protocol of the discipline of psychology. For example, Milgram's (1962) original study on obedience to authority was not even an experiment. There were no systematically manipulated variables or introductory psychology students randomly assigned to condition. Even less obvious, what many recognize as the first published study

of cognitive dissonance processes was an ethnographic study of a doomsday cult (Festinger, Riecken, \& Schachter, 1964).

Several contributing factors helped to nurture our present day toolbased science. The first of these is that scientists tend to follow what has worked in the past, and this tendency is prudent, since scientific discoveries are rare and so when a method appears to be fruitful, it is wise to follow that same method. Therefore, many tools develop a following after the tool has shown promise and convinced other researchers that it is worthy of continued use. Examples of methods that come into their own and continue to be widely used are numerous but a few; the two-group pre-test/ post-test design is one of a few that has stood the test of time across most disciplines in experimental social science. In contrast, Ebbinghaus' (1885/1913) method of serving as his own subject never really caught the attention of other researchers and largely ended with his work.

Second, scientists and researchers are a conservative group who loathe to make reckless changes to their work unless change is clearly mandated. The problem is that changes are rarely indicated and, if indications exist, those indicators are so subtle that most people either ignore them or never detect them. Using randomization to form two equivalent (or nearly equivalent) groups is so widespread that it would require tremendous effort to convince any reviewer that randomization might be contraindicated. Many of us have direct experience with funding agencies turning down proposals primarily on the basis of a failure to randomize. The randomization process is sacred, and violating the sacred traditions of empirical science these days is not only heretical but also results in disciplinary punishment (i.e., rejected papers, grant proposals, contracts, etc.).

Not only are scientists conservative in their methods, they also are conservative with their professional futures. The current United States climate for research is one that favors publication and extramural funding over all else. To enter and remain in the research community, scientists feel compelled -and rightly so -to work on projects that are most likely to be identified and valued by their peers. Peers, after all, evaluate research through the journal and grant review process, and decide if the contribution is worthy of inclusion. The primary method that researchers use to evaluate one another is through reading publications. Additionally, there is an implicit understanding that the publications will follow along 
very tight lines of a research program. Graduate students are frequently warned that if they do not identify with a particular research area -almost without exception a content area -then they will have a difficult time finding a job after graduation. A "content free" researcher is not one who readily finds employment. The incentives for employment and professional advancement, therefore, drive the use of conservative, unimaginative procedures that do little for scientific progress.

Finally, the tools that are so tightly ingrained in our research efforts are available and easy to implement, thus the incentive to use them. Both research design and data analysis succumb to this potential problem. Since experimental designs are so well entrenched in training and publishing, little effort is needed by the investigator to defend the practice. Moreover, most of the research staff (graduate students, project coordinators, data analysts, etc.) are familiar enough with the logic of randomized experiments that little training is needed to conduct the study. Even computer programs exist to assist in the randomization of subjects to experimental conditions. It is even clearer to see how availability and ease of use shape behavior in the realm of statistical analysis. There are few analyses that are easier to conduct with the latest software than an ANOVA. In fact, many statisticians complain that computer programs have made statistical analysis available to everyone, including those who do not know what they are doing or why they are doing it (Rosnow \& Rosenthal, 1995).

The factors that contribute to a tool-based science can thus be understood in terms of both informational and normative influence (Deutsch \& Gerard, 1955; Cialdini \& Trost, 1998). The factors we identified each represent the impetus to conduct science in a manner that is perceived as correct and acceptable. It is not uncommon for researchers to begin their study of a novel hypothesis by combing the literature to see what has been done in the past within the study of similar phenomena. Routines become established to the degree that scientists understand explicitly or implicitly -that the key to success in the lab and the field depends on convincing one's peers that they have conformed to past research by employing the accepted methods of study. We can only speculate about whether researchers use routines because they believe there are only a few "correct" methods for studying behavior (i.e. internalization). However, research on informational social influence (e.g., Sherif, 1936), as well as self-perception (Bem, 1972), suggests that routines will be established and become self-perpetuating when researchers perceive that they have selected the correct tool. Moreover, perceived pressure to conform to the accepted routine can also promote rigid and uncreative thinking (J anes \& Olson, 2000).

Our speculation about the causes of a tool-based science are not offered to find fault with the tools or the scientists who use them. It is far too easy 
to slip into blaming the victim(s) for the situation at hand. Rather, we offer these possible explanations to call attention to the power of normative pressures and routine use as a means of generating methods to reshape behavior and shift researchers' attention toward a potentially more productive approach to scientific inquiry.

By adopting a tool-based approach to science, researchers have constrained dissonance to a circumscribed situation that would exist almost exclusively in a lab and never a natural setting. Thus, there appears to be an inverse relationship between the availability of data and tool use; as the number of studies increase, researchers become more narrowly focused in their questions and more tool-based in their application. As a result, the literature on a certain phenomenon begins to exhibit signs of creeping operationalism, with methods and tools becoming proxies for the effects themselves.

\section{What are tools and routines?}

Social scientists use a great variety of methods as tools in their pursuit of knowledge. To be clear about what we mean by a tool-focused approach, we explain these tools as follows: "tools" are primarily but not exclusively methodological or statistical procedures -the primary means of critical inquiry -used by social scientists. Just as a carpenter relies on several classes of tools to complete the job at hand, a researcher relies on both methodological and statistical tools for research. Methodology tools consist of approaches typically covered in a graduate research design class. Random selection, random assignment, comparison groups, and pre/ post tests are just a few of the tools typically used throughout social science. These tools are used to maximize decreases in uncertainty in empirical research. Without strong methods, our inferences are weak and uncertainty remains unchanged.

Statistical tools are routines applied in data analysis to quantify our uncertainty. Analysis of (co)variance (ANOVA/ANCOVA), hierarchical linear models (HLM), item response theory (IRT), multiple regression and correlation (MRC), and structural equation models (SEM) are just a few of the many statistical tools readily available to contemporary social scientists. Once a researcher has set-up a study that is maximally effective in reducing uncertainty, the statistical procedures provide estimates for how uncertain the consumers of the work ought to be about the phenomena of interest. The methodological tools may be more subtle and less likely to be highlighted in comparison to the statistical tools, but both classes of tools represent the majority of what we refer to as the "toolbased" approach to science.

A third, perhaps more subtle tool comes in the form of heuristics. Many researchers learn simple rules of thumb when designing studies, reporting 
results, or interpreting findings. Rosnow and Rosenthal's (1995) work cited above serves as an excellent example of heuristic tools that may mislead researchers. Another example might be the testing of main effects before interactions in the typical linear model analysis. Heuristics are the specific judgments made with research methodology and statistics, and therefore are not unique.

As we have discussed, the field of psychology, like the other social sciences, is not immune to the routine use of tools. All sciences use tools but most fields have no strong preference for a restricted number. The routine use of a restricted set of tools suggests that there exists some form of disciplinary bias that favors particular tools over others. It is unlikely that even a restricted set of routinely used tools are appropriate for all applications. Moreover, many tools come into widespread use only after a researcher publishes the utility of that tool under particularly favorable circumstances. Once publicized in the appropriate peer-reviewed venues, the tool is gradually adopted and broadly applied well beyond those conditions where its initial utility was illustrated.

The idea of routines we have presented here and those that help to mandate a tool's use beyond its initial utility has essentially three aspects common practice, mandated use and blind application. At least one of these three factors is necessary but may not be sufficient for a practice to be considered routine. For example, researchers may commonly use a computer for statistical analyses but the use of a computer is neither mandated nor thoughtlessly selected. We do not consider the use of a computer in this case to be a routine. In most cases, however, the strength of a routine will be indicated by the presence of all three aspects.

Consider, for example, the use of random assignment in social science as presenting these three aspects. Researchers commonly employ random assignment in their research designs. Those responsible for gauging the scientific merit of a study (e.g., journal editors and their reviewers, and granting agency reviewers) mandate the use of random assignment for strengthening causal inference. Many researchers blindly comply with the implicit mandate by reviewers and accept the common practice of random assignment even when there are contraindications for its application. Many problems in social and behavioral science are simply not amenable to random assignment (e.g., patient preferential treatment selection). However, random assignment has come to be seen as the benchmark for quality research. Non-randomized studies (e.g., field studies, studies in naturalistic settings, and observational studies) often contribute to our understanding of a phenomenon and some potential causes; the strength of those causal inferences is often lower than in randomized studies. Some non-randomized studies ought to be conducted using randomized designs and vice versa. Regardless of the potential contribution of both, we 
contend that random assignment meets all three criteria for what we consider to be a routine.

Journal reviewers frequently and routinely insist on traditional scientific approaches (e.g., laboratory studies, random assignment, frequentist hypothesis testing, etc.) that may not necessarily be appropriate for decreasing uncertainty within an area of inquiry. Some journals only allow for laboratory-based studies; and yet many of those studies could be just as effectively conducted outside the laboratory. Anecdotally, we have received a hundred communications by e-mail or direct correspondence in which a researcher has complained that reviewers insisted on an approach that fit "well" with the journal's expectations but was completely inappropriate for the researcher's question at hand. Two examples serve to make this point. One researcher complained that his study had come under heavy scrutiny because no placebo was used in a randomized treatment trial. The lack of a placebo, in this case, was not only impractical but it was also irrelevant to the scientific question -namely: did an alternative and new treatment outperform the standard treatment? A placebo or inactive treatment group would have put a financial burden on the research project for no reason other than to satisfy the requirements of a tool-based approach.

The second example of this tool-based focus involved the computation of statistical power. A researcher's grant proposal was viewed because it lacked a power analysis. On the surface of this critique, one might assume that a proposal that lacked a power analysis might be a poor candidate for funding, however, the proposal did not include traditional null-hypothesis significance testing (NHST) but instead used a Bayesian approach for data analysis. Power analysis is only applicable if NHST or some other frequentist approach is used. In all other cases, a statistical power analysis is irrelevant. Sample size justification might be a better term but statistical power is the preferred predominant tool. The blind insistence that a power analysis be included in all grant proposals appears to us to indicate an overly heavy reliance on a standard set of tools when proposing research.

These two examples, while anecdotes, are not anomalies. Most, if not all areas across social science tend to be burdened by the over-reliance on a constrained set of tools and constraints put on the field by reviewers or other decision-makers. These decision-makers may accept the tools as standards, lack the understanding and insight into critical inquiry, or be too overburdened to look beyond the standard approaches. Since journal space and extramural research funds remain dear, reviewers do not seek reasons to accept but rather reasons to reject. Failure to comply with contemporary norms offers a readily available reason to reject. 


\section{A problem-focused science}

An alternative perspective to the tool-based approach to science is a problem-focused approach. It is important to note that we do not refer to problems in the sense that they must have broad, practical, and social appeal. Rather, a problem may be as simple as a basic hypothesis of only minute theoretical value with little or no immediate practical use. Basic science is full of important problems with little immediate practical use. If solving a problem, whether basic or applied, is the research focus then no tool-based approach could be suitable for solving all problems. J ust as an engineer faced with designing a car that runs on less fuel is expected to create a vehicle significantly more fuel-efficient than its predecessors, the researcher must be bound to show that the results are consistent with the focal problem. An engineer who develops a car that is attractive, maneuverable, and fast will never convince others that those qualities are more important than reducing fuel consumption. The same applies to the researcher. There must be something gained in each study that decreases uncertainty about a problem.

Two early critics of scientific inquiry provide us with a reasonable framework to build our explanation of a problem-focused science. Chamberlin (1965) offered the perspective that scientists were too heavily invested in their own theories. He urged scientists to assume a more detached role and pursue focused studies with multiple working hypotheses. Multiple hypotheses would counter any favoritism an investigator might have toward one single hypothesis -or at least that is what Chamberlin argued. What makes Chamberlin's work consistent with our discussion is that he never urged scientists to use any particular tool but rather to be focused on the process of problem-solving. Chamberlin focused on developing a technique for debiasing investigators. In addition and perhaps unintentionally, maximizing information gain is the consequence of using multiple working hypotheses. The second critic Platt (1964) -provided more direction to scientists but that direction was to devote efforts to critical tests whereby maximizing uncertainty was the basis for his recommendations. Platt argued that strong inferences via critical tests were rare and needed more attention by researchers. Both Chamberlin and Platt provided perspective on how science ought to be conducted in a very general sense. Conspicuously absent from their contributions are specifics about which tools to use and how to use them. Rather, they provided the groundwork for a problem-focused approach to scientific inquiry.

While these critics were working in the biological and physical sciences, their perspectives are equally appropriate for social science inquiry. In this vein we present two historical examples that illustrate this problem-focused approach quite well. Due to the dearth of specific social- 
science examples, both examples come from medical research. We use these examples not to offer a comparison between medical research and social science research but to highlight the differences between problemfocused inquiry and tool-based inquiry.

The first example of a problem-focused science comes from the discovery of cholera in England. The efforts of J ohn Snow have been retold by numerous authors (e.g., Freedman, 1992) including Snow himself (1936), and his story is quite simple. People were getting sick and dying from a new medical condition in London. The rate of death made the disease a risk not only to every person in London and its surrounding area, but it made it the most important public health problem at the time. The problem, as Snow saw it, was to find the nature and cause of the illness so it might be either slowed or eradicated. Only a true decrease in uncertainty would eliminate the problem. Snow systematically studied the geographical areas affected and unaffected by the illness, and eventually, by luck and critical thinking, reduced the source of the problem to a water well located in one part of London. He identified areas adjacent to one another and studied the cases where only one area was affected. These comparisons served as his methods for determining the relevant variables for further investigation. He performed no formal statistical tests performed and implemented no randomized designs; rather, he used informal mental calculations to track the ratio of sick versus healthy individuals in each area. Snow did not implement any of the procedures that are now mandated in social science. He used purely observational methods and no formal statistical analyses and yet he solved the problem. During Snow's investigation, several other researchers developed theories as to how and why the illness spread, and they used the tools commonly accepted at the time. Unfortunately for the London citizens, none of their competing theories helped, although the researchers were largely applauded for their brilliant insights. Only Snow had the determination to identify the source of the illness. It was his dedication that led to a great medical discovery in pathogenesis and bacterial infection now known as cholera.

The second example comes from a little known study of cancer in rural China. A production script (Goldwyn, 1979) detailing the methods and procedures of the study documents a problem-focused inquiry into the causes of a esophageal cancer. A team of researchers, including epidemiologists, virologists, chemists, geologists, pathologists, and surgeons, investigated an incredibly high incidence rate of cancer in the Lin Xian valley in rural China to determine its cause. One important complication was the fact that no single causal agent could be identified. Instead, multiple causes might operate together to give rise to cancer. A long process interrupted by the Cultural Revolution in China eventually led to the discovery of the fine balance between Vitamin $\mathrm{C}$ and nitrites. If 
the balance was disturbed by environmental distress (i.e., plants growing in molybdenum poor soil causing the nitrite levels to increase and vitamin C to decrease), people living on the plants of the region were at higher risk for cancer. This discovery led to other more significant findings relating to the generation and maintenance of cancerous tissue. All of their findings and progress were directly attributable to their unfailing desire to solve the focal problem. At no time did the investigators employ standard methodological or statistical procedures. Their research is based upon a single sample that a multidisciplinary research team tirelessly and thoroughly analyzed. Because of their efforts, uncertainty was decreased significantly.

\section{Conclusion: The Essential and Non-Essential Components of a Problem-Focused Approach}

While the two examples are illustrative of a problem-focused approach, the presentation does little in the way of helping us prescribe what it means to be problem-focused. There are likely only two essential ingredients that form a problem-focused approach to critical inquiry. The first ingredient is a focal problem to investigate. While this might sound trivial, there exists no shortage of research in social science that fails to state a priori any explicit goal of decreasing uncertainty about a specific problem. What we mean by a problem is an actual theoretically-driven proposition that is testable but untested. The second ingredient that is common to all problem-focused approaches is having a goal. While the problem changes with each new investigation, the goal always remains the same: to decrease uncertainty about a specific problem. We must not confuse the trivial effort of computing correlations between variables out of curiosity with having a goal. The goal follows as a focus to decrease uncertainty about the likelihood of the theoretically driven proposition. What remains non-essential is any fixed formula for attaining the goal.

Our purpose for this article is to draw attention to a subtle trend that might have substantial ill-effects on the progress of science. We simply want to caution that a tool-based approach to research is likely to slow progress and could even halt progress altogether. Furthermore, tool-based science may produce far more journal articles that have little impact on the problems we desire to solve. In addition to offering this perspective, we also offer cues of what constitutes the trend and exemplars of alternative research projects that were not burdened by the tool-based approach. These cues and exemplars hopefully serve as debiasing agents to correct both researchers and reviewers from following research agendas restricted by methodological and statistical tools. Finally, we offer this critique to stimulate interest in the epistemology of social science and invite informed debate from our colleagues. 
Unfortunately, social scientists have drifted away from the roots of empirical research. Bacon's (1620/2000) original guidelines for conducting empirical work include starting with a problem. He reasoned that if a problem were identified and certain observations were taken under specific conditions, our uncertainty about that problem would decrease. In contemporary social science, the order of events is backward. A general question or set of questions guides the research. If the findings are inconsistent with the initial intentions, then the researcher either abandons that effort, since null findings are rarely if ever published, or the researcher generates ad-hoc alternative questions. By reverting back to our empirical roots and adopting a more problem-focused approach, scientific progress may be improved.

Social and behavioral science has moved away from its roots as a problem-focused and solution-oriented endeavor. Research is now a routine. To get funded, published, or even accepted by most organizations, researchers must adopt a routine approach to research that we refer to as a tool-based science. The questions that challenged us a 100 years ago, such as prejudice, consciousness, the function of dreaming, the interaction between genetic predispositions and environmental forces, remain the same today. We are not likely to solve them by merely changing our methods. One thing is certain; an unimaginative routine will not help us solve these problems in any expedient manner. Psychologists ought to strive to solve problems. A problem-focused approach might lead to greater methodological variability and faster progress than we see in contemporary psychological science.

\section{References}

Asch, S. (1956). Studies of independence and conformity: I. a minority of one against a unanimous majority. Psychological Monographs, 70.

Bacon, F. (1620/2000). Novum organon (francis bacon: The new organon) (L. J ardine \&M. Silverstone, Eds.). Cambridge: Cambridge University Press.

Bem, D. (1972). Self-perception theory. In L. Berkowitz (Ed.), Advances in experimental social psychology (Vol. 6, pp. 1-62). New York: Academic Press.

Brunswik, E. (1956). Perception and the representative design of psychological experiments. Berkeley: University of California Press.

Chamberlin, T. C. (1965, May). The method of multiple working hypotheses: With this method the dangers of parental affection for a favorite theory can be circumvented. Science, 148 (3671), 754- 759.

Cialdini, R., \& Trost, M. (1998). Social influence: Social norms, conformity, and compliance. In D. Gilbert, S. Fiske, \& G. Lindzey (Eds.), The handbook of social psychology (4th ed., Vol. 2, pp. 151- 192). New York: MoGraw-Hill.

Cohen, J. (1962, Sep). The statistical power of abnormal-social psychological research: a review. J ournal of abnormal and social psychology, 65, 145- 153. 
Deutsch, M., \& Gerard, H. B. (1955, Nov). A study of normative and informational social influences upon individual judgment. Journal of abnormal psychology, 51, 629-636.

Ebbinghaus, H. (1885/1913). Memory: A contribution to experimental psychology (H. Ruger \&E. Bysseniene, Eds.). New York: Dover.

Festinger, L., \& Carlsmith, J . M. (1959, Mar). Cognitive consequences of forced compliance. J ournal of abnormal psychology, 58, 203- 210.

Festinger, L., Riecken, H., \& Schachter, S. (1964). When prophecy fails. New York: Harper.

Freedman, D. (1992). Statistical models and shoe leather. Sociological Methodology, 22, 291- 313.

Goldstein, W., \& Hogarth, R. (1997). Judgment and decision research: Some historical context. In Research on judgment and decision making (pp. 3- 65). New York: Cambridge University Press.

Goldwyn, E. (1979). China's campaign against a valley of cancer: The cancer detectives of lin xiang. The Listener Magazine, British Broadcasting Corporation (BBC).

Janes, L., \& Olson, J . (2000). J eer pressure: The behavioral effects of observing ridicule of others. Personality and Social Psychology Bulletin, 26, 474- 485.

Meehl, P. (1978). Theoretical risks and tabular asterisks: Sir karl, sir ronald, and the slow progress of soft psychology. Journal of Consulting and Clinical Psychology, 46, 806- 834.

Milgram, S. (1962). Behavioral study of obedience. J ournal of Abnormal and Social Psychology, 67, 317- 378.

Platt, J . R. (1964, Oct). Strong inference: Certain systematic methods of scientific thinking may produce much more rapid progress than others. Science, 146 (3642), 347-353.

Rosnow, R., \& Rosenthal, R. (1995). Some things you learn aren't so: Cohen's paradox, Asch's paradigm and the interpretation of interaction. Psychological Science, 6, 3-9.

Sherif, M. (1936). The psychology of social norms. New York: Harper.

Snow, J . (1936). Snow on cholera. London: Oxford University Press.

Zuckerman, M., Hodgins, H., Zuckerman, A., \& Rosenthal, R. (1993). Contemporary issues in the analysis of data: A survey of 551 psychologists. Psychological Science, 4, 49- 53. 